\title{
ÉDOUARD GLISSANT, UNE PAROLE OUVERTE SUR LE MONDE
}

\author{
ÉDOUARD GLISSANT, UMA FALA ABERTA AO MUNDO
}

Mylène Danglades ${ }^{1}$

Résumé: Édouard Glissant, dans son Traité du Tout-monde paru en 1997 nous propose sa vision d'un monde changeant qui oriente la pensée, le regard humain et génère par-là même une parole ouverte mettant en exergue des identités culturelles inédites. Cette parole cherche à extirper des miasmes de la conscience, du chaos de la mémoire une histoire créatrice. Il s'agit, par le biais de l'écriture, de la langue ou des langues, de percevoir le mystère du vivant ou d'en approcher. Mais l'humain dans "un monde chaos" peut-il se raccrocher à une identité stable en lien avec sa nation, sa langue, sa race, sa religion ou son ethnie? Autrui ne s'inscrit-il pas contre son gré dans ce scénario? La relation à l'autre ne vient-elle pas remettre en question ces fondamentaux, ce principe unique? L'Altérité s'organise autour d'un “je” et d'un "nous, d'un jeu entre ces différentes entités, entérinées ou tenues à distance. La poétique de la relation repose sur une opposition que l'auteur qualifie de "bénéfique", mais si nous sommes plongés dans l'obscurité, en proie à de mystérieuses sensations, mais il importe avant tout d'extraire de tout cela "de nouvelles possibilités créatrices". Mots clés: parole; monde; identité; langue.

Resumo: Édouard Glissant, em seu Traité du Tout-monde, publicado em 1997, propõe-nos sua visão de um mundo em mudança que orienta o pensamento, o olhar humano e gera, assim, um discurso aberto que destaca identidades culturais inéditas. Esse discurso busca extirpar miasmas da consciência, do caos da memória uma história criativa. Trata-se, por meio da escrita, de uma língua ou de línguas, de perceber o mistério da vida ou de acercá-lo. Mas pode o ser humano em um "caos mundial" se apegar a uma identidade estável em conexão com sua nação, sua língua, sua raça, sua religião ou sua etnia? Não se inscreveria ele contra a sua vontade nesse cenário? A relação com o outro não questiona esses fundamentos, esse princípio único? A alteridade é organizada em torno de um "eu" e um "nós", em um jogo entre essas diferentes entidades, endossadas ou mantidas à distância. A poética da relação é baseada em uma oposição que o autor descreve como "benéfica", mas se mergulhamos na escuridão, nas garras de sensações misteriosas importa, sobretudo, extrair de tudo isso "novas possibilidades criativas".

Palavras-chave: discurso; mundo; identidade; língua.

En nommant expressément le nom de l'écrivain martiniquais Edouard Glissant, il n'est pas étrange de voir affluer aussitôt à l'esprit certaines formulations ou images récurrentes. On évoque allègrement les concepts de "l'antillanité", du "Tout- monde", de la "Relation" ou de la "créolisation", comme autant de linéaments pour définir l'auteur. Les mots s'inscrivent dans un système d'échos, de réseaux, nous conviant par là-même selon la formule d'Edouard Glissant à “consentir à l'élan infinitésimal, à l'ajout" (1990, p.59). On peut aussi y entrevoir de la part de l'auteur, un élan, une modulation sensible pour rendre intelligibles des intuitions, des projections. Son œuvre se veut conceptuelle, mais elle dépasse cette limite pour s'étendre ou se répandre au roman, à la poésie, au théâtre et aux essais philosophiques. A travers ses multiples écrits qui se relaient ou s'entrelacent, l'auteur nous propose sa conception d'un monde changeant apte à orienter la pensée et le regard humain. Alexandre Leupin (1998) évoque, à ce propos, cette œuvre

\footnotetext{
${ }^{1}$ Maître de Conférences en Cultures et Langues Régionales. Université de Guyane. Laboratoire MINEA (Migration, Interculturalité et Éducation en Amazonie). E-mail: mylene.danglades@univ-guvane.fr
} 
glissantienne qui "se développe en spirale, ruminant toujours les mêmes matières à penser, se développant jusqu'à couvrir et embrasser une immense étendue de multiplicités vivantes, qui sont le ferment de nouvelles découvertes" (p.41). Dans Poétique de la Relation: Poétique III (1990), Glissant mentionne ce travail "en spirale", cette circularité qui serait à l'œuvre dans nos espaces et qui sous-tendrait toute la création scripturale, toute pratique d'écriture. Les Entretiens de Baton Rouge permettent à Edouard Glissant de se remémorer un texte d'Henri Pichette qui sert de soubassement à sa philosophie. Ce dernier indique que "La littérature n'est belle que dans le lit du monde" (2008, p.54). Cette littérature cherche en profondeur, s'apparente selon Raphaël Lauro (2015) à "une lecture archéologique", basée sur une superposition de textes accumulées au fil du temps. La langue axée sur des images ou des concepts trace les sillons permettant de suivre le cheminement de la pensée.

Dans sa Poétique de la Relation (1969), Glissant esquisse l'idée d'un vieil homme ignorant en matière poétique, mais qui porte en lui la mêlée des terres, des histoires comme s’il s'agissait de "chercher l'espace du monde" à travers des sillons et les emmêlements. L'auteur écrira plus tard que "Dire le monde, c'est faire le monde", comme pour signifier les profondeurs, "les possibles de la voix". Il importe de lui emboîter le pas et de suivre minutieusement la démarche suivante :

Tu dessouches - tu enracines - ce même tronc. Tu écris le même mot. Tant de redites, d'évidences depuis un si long temps, et ces multiples strates de l'écriture, chaque pensée déblayée du terreau, chaque pan déboulé du poème concourent à ce rassemblement. [...] Tissant un arbitraire ou ambitieux lien de l'œuvre future au monde toujours présent, je continue, par ouvrir sur la perspective du monde les deux battants fermés sur l'œuvre: l'entassement des matières, puis leur "signifiance”. (GLISSANT, 1997, p.15)

La matière s'organise à partir des souches, des racines et du terreau pour traduire le monde dans sa pluralité. Il s'agit d'œuvrer en profondeur, de "tisser" une trame accessible dans le temps et dans l'espace. Ce monde évoqué donnera lieu à la notion de "Tout-Monde" et celle-ci deviendra progressivement le titre de son roman datant de 1995. Elle prendra forme et se développera dans son Traité du Tout-monde publié en 1997. Nous pouvons citer quelques titres parmi ses autres ouvrages mettant en exergue le terme "monde", notamment des essais: Une nouvelle région du monde: Esthétique 1(2006); L'Intraitable Beauté du monde. Adresse à Barack Obama (2009) - avec Patrick Chamoiseau. Au niveau de la poésie, signalons La Terre le feu l'eau et les vents: une anthologie de la poésie du Tout-monde (2010). En ce qui concerne le théâtre, retenons le titre suivant: Le Monde incréé: conte de ce que fut la Tragédie d'Askia, Parabole d'un Moulin de Martinique, La Folie Célat (2000).

Son travail d'écriture dépasse la sphère générique et engendre chez lui "une poétique du divers", une "poétique de la Relation" ou une "poésie en étendue" qui ne se borne pas au genre, mais qui réinvestit "une nouvelle région du monde" ou qui se réinvente en permanence. Et lorsqu'on se réfère à Edouard Glissant pour comprendre l'appellation “Tout-Monde", il la définit lui-même en ces termes:

J'appelle Tout-Monde notre univers tel qu'il change et perdure en échangeant et, en même temps, la "vision" que nous en avons. La totalité-monde dans sa diversité physique et dans les représentations qu'elle nous inspire: que nous ne saurions plus chanter, dire ni travailler à souffrance à partir de notre seul lieu, sans plonger à l'imaginaire de cette totalité. Les poètes l'ont de tout temps pressenti. [...] La conjonction des histoires des peuples propose aux poètes d'aujourd'hui une façon nouvelle. La mondialité, si elle se vérifie dans les oppressions et les exploitations des faibles par les puissants, se devine aussi et se vit par les poétiques, loin de toute généralisation [...]. (GLISSANT, 1997, p.176)

L'auteur nous esquisse une "vision" globalisante du monde, un univers qui nous est présenté comme un "tout", tant au niveau des représentations, des imaginaires que de la poétique. La parole cherche à extirper des miasmes de la conscience, du chaos de la mémoire, une histoire créatrice, car le tout-monde est une nouvelle manière de penser et de regarder autour de soi, une parole ouverte qui s'offre au monde. Il s'agit, par le biais de l'écriture, de la langue ou des langues, 
de percevoir le mystère du vivant ou d'en approcher. Mais l'humain dans "un-monde chaos", en proie à un bouleversement perpétuel, peut-il se raccrocher à une identité stable en lien avec sa nation, sa langue, sa race, sa religion, son ethnie ou son semblable de manière générale? La relation à l'autre ne remet-elle pas précisément en question ce principe unique?

L'Altérité s'organise autour d'un "je" et d'un "nous", d'un jeu entre ces différentes entités, entérinées ou tenues à distance. La poétique de la relation repose sur une opposition que l'auteur qualifie de "bénéfique", mais si nous sommes plongés dans l'obscurité, en proie à de mystérieuses sensations, il importe avant tout d'extraire de tout cela "de nouvelles possibilités créatrices".

\section{Le lieu et la parole poétique}

Edouard Glissant au début de son roman Tout-Monde, paru en 1993 rappelle en guise de prologue, les péripéties qui ont précédé et généré des romans écrits depuis 1956, de manière à aboutir à un système d'échos, de résonnances. L'écrivain, dans son acte créateur et le cheminement de sa pensée, intègre des éléments qui s’inscrivent dans une forme de continuité. Les épisodes discontinus du roman, amalgament les temps, depuis les premiers romans et la rencontre entre les békés Laroche et Senglis, propriétaires d'esclaves en 1788, la mort de Longoué, héritier des "marrons" en 1945, les pérégrinations du narrateur en France dans les années 1950-60, jusqu'aux révoltes et à la violence des années 1970 en Martinique. D'étape en étape, on en arrive aux retours et aux détours les plus actuels. En se référant à une étude menée par Dominique Chancé,nous sommes interpellés par un point, à savoircet espace dans la littérature caribéenne qui serait parcouru dans tous les sens. Et dans sa thèse, L'Auteur en souffrance (2000), Chancé poursuit sur cette lancée et évoque la poétique d'auteurs rattachés à divers terroirs,tels Cuba avec Alejo Carpentier, la Guadeloupe en ce qui concerne Daniel Maximin, la Martinique dans le cas d'Edouard Glissant ou Trinidad avec Naipaul, une région homogène, formelle ou uniforme et en même temps une région fonctionnelle ou systémique. Si l'on se réfère aux postulats de Mateo Rodriguez de la Faculté de Géographie de la Habana, cette "région uniforme comporte des aires individuelles relativement homogènes, au sein desquelles prédominent certains types d'espaces et de paysages géographiques" (2013, s/p). Mateo Rodriguez souligne que la région géographique des Antilles se caractérise par

l'insularité et la maritimité, configurées par un arc d'îles complexe, la tropicalité, la grande diversité et la miniaturisation des espaces et des paysages, la présence de populations métisses et d'origine africaine, qui ont humanisé le paysage de façon particulière, avec ses coloris et sa morphologie particulière, le rôle déterminant au départ de la plantation sucrière en conformité avec un modèle spécifique d'organisation spatiale, une domination de la culture des champs de canne et une centralité représentée par les usines à sucre comme élément d'intégration. (RODRIGUEZ, 1989, s/p)

Ce sont les particularités essentielles de la géographie "caribéenne" qu'on peut globalement retrouver dans autres régions. Un réseau de relations fonctionnelles, de flux, de centres émerge et peut tisser des liens souterrains entre toutes ces entités.

Dominique Chance met en lumière que les écrivains, quelles que soient leur origines et leur histoire, cherchent à redonner une identité à une collectivité, à un ensemble archipélique éclaté, complexe. Ce sont les Antilles, au sens large, qui retranscrivent la métaphysique du monde moderne. Cet ensemble très vaste combine la singularité et la pluralité des points de vue. L'humain est rattaché à une sphère, à un "paysage", définie par Le Robert comme la "partie d'un pays que la nature présente à l'œil qui la regarde", et dès lors, ce paysage empreint des paramètres subjectifs, émotifs et affectifs se donne à lire "en dessous", dans une verticalité profonde. Il ne s'agit pas d'accaparer ou de dominer les éléments, mais de les laisser exister ou irradier autour de 
soi par le biais de la parole. Le discours heideggérien (2009) souligne d'ailleurs le rapport entre l'homme et l'espace qui se concrétise dans le fait de d'essarter, [de] dégager, [de] donner du champ-libre, de l'ouverture. La poésie, autrement dit, l'acte créateur, unificateur entre l'homme et la terre constitue en soi l'essence profonde de l'habitation humaine, ce qui le conduit à une éventuelle projection, une nouvelle temporalité et spatialité pour l'humanité, à "une nouvelle région du monde" (GLISSANT, 1958). Selon Heidegger, "[La poésie] ne survole pas la terre, elle ne la dépasse pas pour la quitter et planer au-dessus d'elle. C'est la poésie qui tout d'abord conduit l'homme sur terre, à la terre, et qui le conduit ainsi dans l'habitation" (1958, p. 230). L'habitation de l'homme revêt une dimension poétique et maintient ce dernier dans une certaine incertitude, une étrangeté à la terre qui l'amène à être partagé entre deux mondes, le céleste et le terrestre. L'homme poétique participe à la naissance et au devenir des choses à travers le langage. Nous pouvons nous reporter à que ce Glissant a écrit dans l'Intentionpoétique sur la poésie contemporaine et la nécessité pour elle de rentrer "dans" les choses, de dépasser toute séparation entre l'homme et le monde, toute représentation intellectuelle et réelle: 'qu'on ne s'arrête pas à l'apparence des choses, au phénomène, au rouage; qu'on entre dans les choses" (GLISSANT, 1969, p.105). Il souligne l'importance d'aller au-delà de l'indicible, du chaos, de la démesure. Habiter le monde en poète équivaut également pour Glissant à un mouvement d'errance, d'expatriation et de rencontre avec l'altérité. Mais cette notion d'altérité fait émerger en parallèle la relation coloniale aux Antilles et le processus de transformation de soi qui en découle.

Alain Ménil (2011, p. 20) évoque, à ce propos, une écriture qui s’inscrit dans un mouvement abyssal "d'ex-centration", refusant tout principe centriste pour se focaliser sur l'expérience du Divers permettant de saisir sa propre singularité, "d'arpenter le Monde - le tout et le divers du monde, et de le saisir tel, constant et dissemblable".

Conscient très tôt des moyens qui sont les siens, l'entrée en littérature de Glissant se fait sous le signe peu équivoque d'une prise de parole qui interdit précisément toute familiarité. L'excentricité est donc bien chez Glissant affaire de position. [...] Elle se tient au croisement du commun et du singulier, dans ce dialogue parfois possible, souvent tendu, que tentent de maintenir la communauté et l'individu, qui ne se noue qu'elliptiquement au point mystérieux où le commun de la communauté, n’imposant pas silence au singulier, permet à sa parole d'advenir. (MÉNIL, 2011, p.32)

Le travail d'écriture s'insère dans un processus de transformation et de subjectivation qui nécessite un positionnement, à savoir une excentricité, un éloignement du pouvoir politique décisionnaire pour s'ouvrir à l'autre, se construire par le biais de la confrontation, de la parole poétique apte à écouter la terre, à participer à la venue au monde du monde. L'homme d'après Glissant doit s'espacer de son propre monde en se focalisant sur d'autres mesures et pour reprendre la formule de Segalen, il importe de "se faire exote", de se soumettre à l'empire du Divers que le voyageur ou l'observateur ne peut appréhender qu'en abandonnant son centre de référence et son appareillage conventionnel de notions et perceptions.

Le temps et l'espace doivent converger pour permettre à l'homme de se retrouver et, dès lors, "l'ailleurs" et "l'ici" (GLISSANT, 2006, 104) deviennent des données caduques, dans la mesure où ce dernier est projeté dans un chaos-monde, unfatras originel, linguistique, culturel et existentiel. Edouard Glissant met en avant un "jointure entre toutes ces conceptions de temps, du mythe, de l'être comme étant, des cultures [...]" (GLISSANT, 1990, p.124). La question du temps ramène l'homme à lui-même et à son "rapport avec les choses" (p.471), à cette oscillation entre la "profondeur" - en soi et depuis son ici originel (le "lieu de naissance au monde", précise Glissant") et "l'étendue", qui appelle chaque destin à un espacement vers l'autre et l'ailleurs, ou, mieux, à cette expérience de "l'altréité" qu' Édouard Glissant désigne "non [comme] la pensée de l'autre mais [comme] l'autre de la pensée" (2006, p.104): "Le poète tâche à enrhizomer son lieu dans la totalité, à diffuser la totalité dans son lieu et son lieu dans la totalité: la permanence dans l'instant et inversement, l'ailleurs dans l'ici et réciproquement" (p.122). 
Le romancier explore le monde parcouru sans endroit ni envers, rapprochant les personnages, les histoires et les langues: "Le langage est un voyage et vous voyez qu'il n'a pas de Fin".Montaigne dans ses Essais souligne déjà que le temps est fait, pour lui, de ces passages qui s'additionnent et qui, dans leur tracé discontinu, n'en constituent pas moins, à l'infini, une harmonieuse continuité. En évoquant ces "passages", soulignons qu'Edouard Glissant s'amuse lui aussi de la circulation des hommes dans le monde. Au moment où Freud énonce que le sujetest radicalement divisé entre sa conscience et son inconscient, Edouard Glissant formule la pensée de sa totalité. Il déclinera la notion de Tout-Monde dans un roman et un essai et notera: "J'appelle Tout-monde [...] la Totalité-monde dans sa diversité physique et dans les représentations qu'elle nous inspire" (GLISSANT, 1997, p.176). Dans le roman Tout-Monde, l'univers des hommes nous est présenté comme une matière poétique relevant de la vision, du rêve et permettant par là-même une jonction entre le sensible et l'intelligible. L'essai intitulé Traité du Tout-Monde est axé sur la théorie. Alexandre Leupin précise à juste titre qu'il s'agit "d'abord [de] la connaissance et [de] la mise au point des inextricables (lieux, paysages, moments), puis la réflexion qui extrait ce qui peut être traité des inextricables" (LEUPIN, 1998, p.107). La forme ouverte de l'essai répond à l'exigence de la reprise, de la recherche, avec ses tentatives renouvelées, les moments de la pensée, successive et variable, de ses diverses combinaisons dans le temps. Le terme "essai", traduit à la fois un acte et une forme, un effort de la pensée s'appliquant à divers objets, et l'esthétique de cette recherche.

On assiste à une évolution de la formule du Tout-Monde, de la forme romanesque à l'essai, comme si l'exercice s'apparentait à une rotation, pour trouver un équilibre entre la mention abstraite et le matériau et entraver ainsi toute forme de dissolution. Dans Tout-monde, Edouard Glissant développe le Divers, l'Etant face à l'Être, l'universel généralisant. et la matière ne se désagrège pas. Dans les premières pages du roman, l'auteur convoque le pouvoir de la pensée et de l'imaginaire pour appréhender l'existence du Tout-Monde réel. Il évoque:

Un pays où la dérive de 1 'habitant, ce par quoi il tient à la terre, comme une poussière têtue dans l'air, c'est cet aller tout aussi bien que ce revenir, à tous les vents. Notre science, c'est le détour et l'allervenir. Un pays ouvert, mais qui ne fut jamais déboussolé de son erre et d'où, si la pensée s'envole, ce n'est pas en fuligineuses déperditions. Un pays éperdu dans ses calmes plats, mais qui ne s'est jamais perdu. C'est de ces sortes de pays-là qu'on peut vraiment voir et imaginer le monde. (GLISSANT, 1993, p.18)

Les termes “dérive”, “déperdition”, “éperdu”, “perdu”, “aller”, "revenir” et "l'aller-venir" s'enchaînent comme s'il s'agissait de souligner l'existence d'un mouvement perpétuel, d'un voyage dans le langage. Et ces éléments génèrent un roman qui obéit à une trajectoire, qui s'enroulent et se ré-enroulent en déplaçant des images et des lieux communs. L'image du cercle se dessine et nous remet en mémoire la définition de la philosophie hegélienne: "La philosophie étant une totalité a, comme telle, son commencement partout. Or, essentiellement, ce commencement est partout un résultat. Il faut concevoir la philosophie comme un cercle revenant sur lui-même (HEGEL, 1979, p.17)”. Glissant inscrit également sa pensée, son langage et son écriture dans un cercle, une dynamique en mouvement pour traduire la totalité.

\begin{abstract}
Alors encore vous entendez ces langages du monde qui se rencontrent sur la vague le mont, toutes ces langues qui fracassent l'une dans l'autre comme des crêtes de vagues en furie, et vous entreprenez, tout un chacun applaudit, de bondir d'une langue dans l'autre, ça fait de grosses dévirades d'imprévu, puis vous coulez dans ce mystère des mots et voilà que vous quittez la débandade et vous filez comme une rivière à sec dans le secret de son partage de rivière [ ... ]. (HEGEL, 1979, p.20)
\end{abstract}

Tout se meut, interagit dans une territorialisation conquérante. Les éléments naturels semblent se déchaîner et entraîner l'homme dans une mouvement ascendant et descendant, des tours et des détours. Le monde entier nous parle, "ce monde [qui] est là, là autour" (p.24), "ce 
hélé du monde ouvert sur vos têtes et sous vos pieds" (p.23), le monde qu'on voit "d'à partir de n'importe quel pays [ ... ]" (p. 24), "Il vous brandit que c'est partout déréglé, déboussolé, décati, tout en folie, le sang le vent, mais que c'est le monde entier qui vous parle par ma voix de Panoplie. 'Ou que vous tournez, c'est désolation. Mais vous tournez pourtant” (p.26). L'homme n'est point statique, figé, il est pris dans une spirale, il doit "aller plus au fond, c'est le plein-style du Tout-monde". Et Edouard Glissant d'ajouter: "Parce ce qu'il vous parle ainsi, c'est le monde" (1979, p.26).

Les personnages symbolisent la diversité. Ils ont dépassé les limites et les frontières, ils mélangent les langages, transbahutent, tombent dans la folie du monde, on les refoule et les exclut de la puissance du Territoire mais, ils sont la terre elle-même, ils vont au-devant de nous, ils voient, loin devant, ce point fixe qu'il faudra dépasser une fois encore. Ce roman est une anthologie de toutes les sortes de voyages possibles. Pino, en s'adressant à Amina et en considérant le ciel et les étoiles, affirme en parlant de Matthieu Béluse: "Il est dans le monde, il est dans le monde entier, là où le temps s'est égaré" (p.48).

Cette coprésence nouvelle des êtres et des choses, cet état de mondialité dans lequel règne la Relation ne peut se concevoir sans un lieu de rencontre de toutes les cultures, ce "tiers-espace" où s'estompe tous les préjugés, selon la formule de Homi Bhabha, utilisée dans son ouvrage Les lieux de la culture (2007). Une théorie postcoloniale. Edouard Glissant évoque "l'étendue, la multiplication du rhizome", "une sorte de prescience du Tout-monde, et qui 'prendraient contact' à l'infini avec toute chose recommencée, en bousculade et en grand plaisir" (1996, p.63).

"La verticalité prodigieusement descendante du banyan", pour se référer au poème de Saint-John Perse (1946), ce banyan "qui avait joint l'élan à la racine", nous rappelle l'étendue, l'espace du monde, "la spectaculaire poussée de la pensée du rhizome" (GLISSANT, 1993, p.63) ou "Les banians [qui] crépitaient en rhizomes dans l'espace du monde", qui "donnaient sens aux équivoques" (p.64). La multiplicité des voix à l'image des racines proliférantes mettrait en exergue des identités culturelles. Elle chercherait à générer desmiasmes de la conscience, du "chaosmonde", de la mémoire, des forces vivifiantes et inventives.

\section{Une mondialité salvatrice?}

Le monde se donne à voir et à vivre dans l'espace-temps, un espace fragmenté qui peut se révéler chaotique, constitué de détours et d'entrelacements, un chaos présenté néanmoins comme quelque chose de "beau", générateur de vie future:

Comme est beau l'enlacement de végétation qui multiplie sans fin dans les détours de la Trace en Martinique, entre Balata et Morne Rouge, ou la volée d'écumes qui s'enroule en éventail fou dans ce moment étincelant ou deux vagues de mer écrasent leurs crêtes l'une contre l'autre. (GLISSANT, 1993, p.62)

La nature, aussi effroyable puisse-t-elle paraître, est à même d'engendrer des figures emblématiques, symboliques d'un sempiternel renouveau. Le tout-monde fait émerger du chaos un mouvement de compression, de spirale cyclique, un regard et un langage neufs, un "déparler" qui contraste avec le discours habituel et policé. Le "tout-monde" ne s'apparente pas à une simple réflexion sur l'univers et l'universalité; il suggère un renversement des tendances, un verbe qui s'offre au monde au même titre qu'une parole ouverte, une planche salutaire. Les langues, comme un bruissement de feuillages au sommet des cimes, atteste de la diversité ou de la "diversalité" des humains, selon la formulation de Glissant. Matthieu essaie de faire comprendre en ces termes le monde à Amina: "La profondeur de la terre est dans son étendue, et sa hauteur chemine. Non, non, allez, n'ouvrez pas les yeux, imaginez toujours. La terre est un Chaos, le Chaos n'a ni haut ni bas, et le Chaos est beau" (GLISSANT, 1993, p.61).

Dans le chapitre relatif à Bezaudin, ce dernier s'adresse en ces termes à Longoué: "tout ce 
falbalas de terrain que vous affirmez que j'ai descendu de travers, en travers de la Martinique, depuis Bezaudin jusqu'au Lamentin, avec ce méli-mélo de branches de vent de courants d'eau de roches de boue" (GLISSANT, 1993, p.206).

La "roche", cette matière argileuse ou ce bassin sédimentaire semble s'ériger comme un socle fondamental pour la renaissance du peuple. Et Longoué de rétorquer à Matthieu:

\begin{abstract}
N'avez-vous pas songé qu'un jour vous allez détracer le Tout- monde et encontrer combien de pays et les parcourir dans leur paysage et leur figuration et les mettre ensemble pour apprendre enfin comment la terre vient dans l'eau et le soleil dans la nuit?

Et est -ce que vous aurez connu le temps qui a venté sur vous, tant que vous n'avez pas mêlé ces pays? Ah bon, dit carrément Matthieu, je ne pensais pas, ou bien plutôt l'idée ne m'était pas venue que je pouvais penser, que voyager au loin pour, comme vous dîtes, mélanger des pays, vous procurait la connaissance du temps qui passe. (GLISSANT, 1993, p.207)
\end{abstract}

L'homme avance au bord du monde, s'y rattache, s'y projette et le temps comme un fils lui permet de lier, de délier les éléments, de lire à travers le passé pour se porter en avant, toujours plus "loin". "Le pays de mer et le pays de terre" (p.207) se raccordent et comme s'il s'agissait d'un enseignement crucial à délivrer, Longoué rajoute:

Parce que le Tout-monde, c'est le monde que vous avez tourné dans votre pensée pendant qu'il vous tourne dans son roulis. Qui donc dit, le monde où vous cherchez cette personne qu'à la fin vous allez rencontrer dans son partage, est-ce une femme est-ce un homme, un vieux corps un enfant. Et le Toutmonde est ce monde-là, où vous cherchez sans partage. (GLISSANT, 1993, p.207)

Il continue en ces termes en employant deux appellations, "l'ombre" et "la lumière" qui semblent particulièrement s'amalgamer:

Ce n'est pas la dernière fois, chanta Longoué, que vous montez dans l' obscur. Attendez donc. Vous le rencontrerez sur toutes les routes, venant repartant. Et si vous ne gravissez pas dans l'obscur, alors vous n'entrerez pas dans la lumière du Tout-Monde.(GLISSANT, 1993, p.208)

Glissant souligne l'importance de l'imaginaire, de la multiplicité des voix, le chemin d'accès au monde créolisé, multilingue et populaire, au Tout-monde:

Là où les systèmes et les idéologies ont défailli, et sans aucunement renoncer au refus et au combat que tu dois mener dans ton lieu particulier, prolongeons au loin l'imaginaire, par un infini éclatement et une répétition à l'infini des thèmes du métissage, du multilinguisme, de la créolisation. (GLISSANT, 1997, p.207)

Un renversement des pratiques s'impose pour permettre à l'homme de s'affranchir d'un certain dogmatisme et de se laisser emporter par le roulis de l'imagination et de la langue. Il est appelé à s'ouvrir au monde. Au-delà de la rotation tourbillonnaire du Tout-monde, l'homme doit s'acheminer vers d'autres modes de pensée, accéder mentalement à de nouveaux territoires, dépasser les procédures intellectuelles ordinaires. Edouard Glissant entrevoit une issue salutaire par le biais "d'une énorme insurrection de l'imaginaire qui portera les Humanités à se créer" (GLISSANT, 2005, p.22-23): "Si nous voulons saisir les principes d'une telle diversité, tressée comme dans un panier, mêlée, il nous faut rassembler tous les possibles de la connaissance et les soumettre à la puissance convergente de l'intuition. L'analyse traditionnelle ne suffira pas ici”' (p.22-23).

La voix du peuple est énoncée comme une vérité qui se doit d'être entendue par tous. Plongé dans le "chaos", la pensée nécessiterait une organisation rigoureuse, mais Edouard Glissant, lui-même affirme que pour "sortir de ce chaos", l'homme doit voir comment "tourner" et évoluer avec le chaos pour "l'habiter et le rendre vivable. L'imaginaire de la créolisation tendrait à inscrire l'homme dans ce processus par le biais d'une langue imbriquée à une autre et difficile- 
ment dissociable. Mathieu, entouré de ses compagnons de jeu recourt au créole et pour les désarçonner, il leur lance: "Les langues créoles sont encore plus créoles que les autres, plus créoles que ce que vous imaginez, nous pouvons vous étourdir avec" (GLISSANT, 1993, p.59). Et devant l'incompréhension de ces derniers, il ajoute: "Ah! Nous pouvons vous faire passer des gros mots pour des inventions, et des traductions littéraires pour du style, et vous restez ébahis! Ces langues créoles sont trop malignes pour vous!” (p.59).

Edouard Glissant, par l'entremise de son personnage, met en exergue un processus de cultures, de "Relation". Un chant vibrant, empreint de résonances multiples et de sensibilité jaillit des entrailles humaines et pense autrement le monde environnant. La langue se fraye une voix et une voie parallèles pour signifier le monde. Le tout-monde inventé par Glissant est basé sur un va-et-vient de la pensée, des histoires entremêlées et énoncées de façon polyphonique. La vie s'écrit et s'énonce, autorise des brassages d'identités, de cultures, des interférences, des symbioses et des heurts. Ainsi, Longoué, personnage du roman Tout-monde clame haut et fort la définition du tout- monde:

\footnotetext{
- Mais le monde n'est pas le Tout-monde dit Longoué.

- Ah ha.

- Ah-ha. Parce que le Tout-monde, c'est le monde que vous avez tourné dans votre pensée pendant qu'il vous tourne dans son roulis (GLISSANT, 1993, p.208).
}

Le roman Tout-monde, publié en 1993, anticipait le Traité du tout-monde, et s'érigeait ainsi sur un imaginaire, une pensée, un récit rendu vivace par les diverses turpitudes des personnages. Le roman donne à penser le monde, sa créolisation, et la rédaction de l'essai par la suite. Toutes les données exploitées ou réexploitées émergent de l'histoire esclavagiste. Et si l'on se réfère au "Traité du Tout-Monde", d'Edouard Glissant, titre qui ne semble point anodin en soi, les "traités" sont directement issus de la traite, comme s'en avise un dénommé Panoplie-Philosophe, déparleur du roman Tout-monde: "Qu'est-ce que c'est un Traité ? exhorte-t-il. Traité de quoi? Traité du bas-monde! Ne récitez plus les Traités! Qu'est-ce que nous sommes. Des maltraités ...”. La parole en étant énoncée, crée et amène des profondeurs à la surface certaines vérités enfouies ou tues. Glissant définit la mondialité comme "une dynamique échevelée du Tout, [...] une haute pensée du monde, que j'appellerais [précise-t-il] non pas la mondialisation (c'en est même le contraire) mais la mondialitée" (2005, p.22-23). Il ajoute que : "La mondialité, ou sens, ou poétique de la diversité solidaire” (2005, p.22-143) est “[...] une aventure sans précédent qu'il nous est donné à tous de vivre, dans un espace-temps qui, pour la première fois, réellement et de manière foudroyante, se conçoit à la fois unique et multiple, et inextricable" (2005, p.23). Il s'agirait pour lui de "[...] penser et agir dans cet inextricable du monde, sans le réduire à nos propres pulsions ni intérêts, individuels ou collectifs, et surtout, à nos systèmes de pensée” (2005, p.24). Mais dans un monde en perpétuelle évolution, et puisque la mondialité repose sur bon nombre de concepts, tels que:

La pensée archipélique convient à l'allure de nos mondes. Elle en emprunte l'ambigu, le fragile, le dérivé [...] Est-ce là renoncer à se gouverner ? Non, c'est s'accorder à ce qui du monde s'est diffusé en archipels précisément, ces sortes de diversités dans l'étendue, qui pourtant rallient des rives et marient des étendues. Nous nous apercevons de ce qu'il y avait de continental, d'épais et qui pesait sur nous, dans les somptueuses pensées de système qui jusqu'à ce jour ont régi l'Histoire des Humanités, et qui ne sont plus adéquates à nos éclatements, à nos histoires ni à nos non moins somptueuses errances. La pensée de l'archipel, des archipels, nous ouvre ces mers. (GLISSANT, 1997, p.31)

Ne sommes-nous pas en droit de nous demander si ces divisions fécondes ne risquent pas de d'engendrer de nouvelles antinomies? Dans La Cobée du lamentin, et plus particulièrement dans son chapitreintitulé "Les Empires qui se suivent et ne se répètent pas", Glissant évoque un nouvel impérialisme qui pourrait éclore vis-à-vis de la mondialisation. Un empire peut chercher à asse- 
oir sa domination sur la Totalité-monde. Glissant objecte:

Le Tout-monde serait la seule force de préservation contre un tel Empire, puisque sa diversité et sa totalité réalisées obligeraient celui-ci à confondre les unes dans les autres ses actions en flèche et ses actions de circularité, l'extension et la préservation, la conquête et la survivance, une éternelle Conquista mêlée à une éternelle Reconquista, installant le trouble et l'ambiguité dans la conception qu'il se serait faite de lui-même. (GLISSANT, 2005, p.154-155)

L'existence du Tout-monde est appelée à contrer le Tout-empire. Mais, cette issue ne dépend-t-elle pas fondamentalement de l'humain? "L'Empire ou le Tout-monde, la balance est devant nous"souligne Glissant (2005, p.162). Les Humanités contemporaines sont-elles sensibles à cette parole ouverte et offerte au monde? Y entrevoient-elles une planche salutaire pour controverser les limbes ou les processus du futur en gestation?

\section{Conclusion}

L'écriture et la théorie forment un tout chez Glissant: elles ne coexistent pas séparément. Elles se relayent annihilant la séparation des genres littéraires et les modes d'écriture. Certains de ses écrits sont rattachés à la philosophie, en l'occurrence la poétique, l'esthétique ou à des formes institutionnelles, telles le discours, l'essai, ou le traité. L'œuvre de Glissant conteste les frontières qui génèrent à leur tour des divisions infinitésimales. Un système de correspondances s'établit d'un ouvrage à un autre et paradoxalement la parole se cherche. Elle menace de tomber dans l'abîme ou se soustrait aux généralités.

Le "tout -monde" n'apparaît pas comme un système parcellaire, claustral, mais comme une parole ouverte sur le monde et offerte à tous les peuples qui parlent des langues minorées. Il évoque "l'identité comme rhizome, allant à la rencontre d'autres racines" (GLISSANT, 1997, p.196). Il s'agit d'un vecteur de diversité et de multiplicité, un point de ralliement, de redéploiement de souffrances partagées générant en retour une somme infinitésimale d' histoires.

Et si les mots s'enroulent et se déroulent à l'infini, si les histoires sont "sempiternellement reprises" (GLISSANT, 1993, p.316), nous pourrions reprendre les propos d'Edouard Glissant et dire: "L'ombrage est favorable pour la parole "et que le" livre n'est pas fini tant que nous n'avons pas trouvé dans un pays inconnu quelqu'un qui s'assied là et qui le récite mot à mot" (p.232) ou comme Panoplie, il vaut mieux "penche[r] vers la terre, pour écouter l'obscur" (p.233). Nous comprenons donc qu'il faut apprendre à lire le chaos-monde. Il s'agit de l'expérimenter dans sa vitalité et son imprévisibilité. Edouard Glissant construit une relecture du mouvement du monde à partir de la refondation d'un paradigme dynamique qui perçoit l'univers comme étant principalement relationnel et résolument antisystème et qu'il dénomme la "Relation"; celle-ci serait à la base d'une forme d'identité en changement perpétuel grâce à la rencontre des différences parce que, dans la "Relation", la différence est à la fois identité et différence. Et sur ce point, Glissant s'accorde à dire que "Nous connaissons que la trace est ce qui nous met, nous tous, d'où que venus, en Relation" (1997, p.18). Il ajoute également ces mots qui montrent qu'il n'est nullement question d'un enfermement: "La pensée de la trace confirme le concept comme élan, le relate : en fait le récitatif, le pose en relation, lui chante relativité" (1997, p.83).

L'être ne se perd pas dans une dilution standardisée" (1997, p.192). "La créolisation ne conclut pas à la perte d'identité, à la dilution de l'étant. Elle n'infère pas le renoncement à soi. Elle suggère la distance (l'en-aller) d'avec les figements bouleversants de l'Etre" (1997, p.25). L’homme se tourne vers le monde et peut scander ces paroles d'Aimé Césaire:

Je me suis, je me suis élargi - comme le monde et ma conscience plus large que la mer.

J'éclate. Je suis le feu. Je suis la mer.

Le monde se défait. Mais je suis le monde. (CÉSAIRE, 1942, p.140)

https://periodicos.unifap.br/index.php/letras

Macapá, v. 9, n. 2, $2^{0}$ sem., 2019 
Aimé Césaire à l'image de bon nombre de poètes et tout comme Glissant est "un rêveur de monde". Il s'ouvre au monde et dans une phase de réciprocité, le monde s'ouvre à lui. Le philosophe Gaston Bachelard a d'ailleurs évoqué dans La poétique de la rêverie (1960) cette "rêverie cosmique" suscitant un syncrétisme entre la représentation et l'unité du monde. Les mots et les images cosmiques relient l'homme au monde.

Somme toute, Edouard Glissant définit ainsi la tâche suprême du poète:"Dire son entour, son pays: dire l'Autre, le monde" (GLISSANT, 1997, p.252). "Le poète tâche à enrizhomer son lieu dans la totalité, à diffuser la totalité dans son lieu [...] l'ailleurs dans l'ici et réciproquement" (p.122). Nous, habitants de "pays ouvert", nous sommes également conviés à lui emboîter le pas et à reconnaître que "notre science, c'est le détour et l'aller-venir" et que "si [notre] pensée s'envole, ce n’est pas en fuligineuses déperditions” (GLISSANT, 1993, p.18).

\section{Références}

BACHELARD, Gaston. Poétique de la rêverie. Paris: PUF, 1960.

BHABHA, Homi. Les lieux de la culture. Une théorie postcoloniale. Paris: Payot, 2007

CÉSAIRE, Aimé. Les armes miraculeuses. Canadá: LEPETITLITTÉRAIRE.FR, 2014.

CHANCÉ, Dominique. L'Auteur en souffrance: essai sur la position et la représentation de l'auteur dans le roman antillais contemporain (1981-1992). Paris: Presses Universitaires de France, 2000.

GLISSANT, Édouard. Une nouvelle région du monde: esthétique I. Paris: Gallimard, 1958.

. L'intention poétique. Paris: Éditions du Seuil, 1969.

Poétique de la relation: poétique III. Paris: Gallimard, 1990.

Edouard Glissant. Tout-Monde. Paris: Gallimard, 1993.

.Traité du Tout-Mond: le cri du monde. Paris: Gallimard, 1997.

Traité du Tout-Monde: poétique IV. Paris: Gallimard, 1997.

Le Monde incréé: conte de ce que fut la Tragédie d'Askia, Parabole d'un Moulin de Marti-

nique, La Folie Célat. Paris: Gallimard, 2000.

. La Cohée du Lamentin: poétique V. Paris: Gallimard, 2005.

Une nouvelle région du monde: esthétique 1. Paris: Gallimard, 2006.

Les entretiens de Baton Rouge. Paris: Gallimard, 2008.

. L'Intraitable Beauté du monde : adresse à Barack Obama. Paris: Galaade, 2009.

. La Terre, le feu, l'eau et les vents: une anthologie de la poésie du Tout-monde. Paris : Galli-

mard, 2010.

HEGEL, Georg Wilhelm Friedrich. Esthétique. Paris: Flammarion, 1979.

HEIDEGGER, Martin. Essais et conférences. Paris: Gallimard, 1958.

Remarques sur art - sculpture - espace. Paris: Editions Payot et Rivages, 2009.

LEUPIN, Alexandre. Édouard Glissant philosophe, Héraclite et Hegel dans le Tout-Monde. Paris: Editions Hermann, 1998.

MÉNIL, Alain. Les voies de la créolisation: essai sur Edouard Glissant. Paris: De L'Incidence Editeur, 2011.

PERSE, Saint-John. Pluies: le banyan de la pluie. Paris: Gallimard, 1945.

RAPHAËL, Lauro. Édouard Glissant et la poésie en étendue: Archéologia d'une notion. 2015. Disponível em: <https://mondesfrancophones.com/dossiers/edouard-glissant/edouard-glissant-et-lapoesie-en-etendue-archeologie-dune-notion/>. Acesso em: 21 out. 2019.

RODRIGUEZ, José M. Mateo. Qu'est ce que la Caraïbe ? Vers une définition géographique.. 2013. Disponível em: <http://www.caribbean-atlas.com/fr/thematiques/qu-est-ce-que-la-caraibe/quest-ce-que-la-caraibe-vers-une-definition-geographique/>. Acesso em: 21 out. 2019.

Envio: 30/10/2019

Aceite: 08/11/2019 